\title{
Assessment of gait in patients with fibromyalgia during motor and cognitive dual task walking: a cross-sectional study
}

\author{
Goran Radunović ${ }^{1}$, Zoran Veličković ${ }^{1}$, Melanija Rašić ${ }^{1}$, Saša Janjić ${ }^{1}$, Vladana Marković ${ }^{2}$ and Saša Radovanović ${ }^{3^{*}}$ (B)
}

\begin{abstract}
Background: The aim of the study was to assess gait pattern of patients diagnosed with fibromyalgia (FM) while performing demanding motor and/or cognitive dual tasks while walking. Further, idea was to explore possible correlations of dual task gait pattern alterations to patients' functional status and presence or absence of clinical symptoms associated with FM.

Methods: Twenty-four female FM patients and 24 healthy female subjects performed a basic walking task, a dual motor, a dual mental (cognitive) and a combined, dual motor and cognitive task simultaneously. Quantitative spatial (stride length) and temporal (cycle time, swing time and double support time) gait parameters were measured using GAITRite walkway system and their variability was assessed. Patients underwent clinical examination including assessment of functional status, pain and fatigue level, psychiatric and cognitive manifestations.

Results: The motor, cognitive and combined dual tasks affect gait performance in FM patients. Difference in tasks between FM and healthy subjects was found as double support time prolongation. Comparison of tasks showing that cycle time in FM was longer than controls and stride length was shorter in patients for all conditions, while no changes were found in any of the gait parameters variability. Further, mental/cognitive dual tasks had a larger effect than motor tasks. Correlations were also found between depression and functional status of the patients and the gait parameters.

Conclusions: Gait is affected in FM patients while dual task walking. No changes in stride-to-stride variability point that patients preserve stability in complex walking situations. Analysis of gait may provide additional information for the FM identification based on presence of clinical features and cognitive status. Correlation of dual task gait alterations with occurrence of clinical symptoms and influence of cognitive changes on gait pattern could additionally define FM subgroups.
\end{abstract}

Keywords: Cycle time, Stride, Gait variability, GAITRite walkway system

\section{Introduction}

Fibromyalgia (FM) is a chronic incapacitating disease characterized by multi-focal, widespread pain. Spectrum of the disease has been broadened to include symptoms

*Correspondence: sasar@imi.bg.ac.rs

${ }^{3}$ Institute for Medical Research, University of Belgrade, Dr Subotića 4, 11129 Belgrade, Serbia

Full list of author information is available at the end of the article such as tenderness, fatigue, non-refreshing sleep, musculoskeletal stiffness, cognitive dysfunction, mood disorders, poor balance, muscle weakness, functional impairments and disability [1,2]. Establishing correct diagnosis is still challenging due to the lack of objective clinical or laboratory markers, with many patients waiting up to 5 years for the diagnosis. To minimize the subjectivity of clinical judgment, several diagnostic criteria were elaborated [3-5]. original author(s) and the source, provide a link to the Creative Commons licence, and indicate if changes were made. The images or other third party material in this article are included in the article's Creative Commons licence, unless indicated otherwise in a credit line to the material. If material is not included in the article's Creative Commons licence and your intended use is not permitted by statutory regulation or exceeds the permitted use, you will need to obtain permission directly from the copyright holder. To view a copy of this licence, visit http://creativecommons.org/licenses/by/4.0/. 
Gait is under strong input of cognitive functions, particularly attention and executive function [6, 7]. In addition to clinical features $[8,9]$ and biomarkers [10], gait analysis was even proposed as an objective measurement for quantifying and subgrouping FM patients $[11,12]$. It was shown that gait is affected in FM patients during dual walking tasks [13-17]. In studies measuring gait while FM patients performed several motor tasks [14, 15], or during base walking [17-19] and walking with different velocities [16, 17], patients showed significant differences in spatio-temporal gait parameters [16-19] compared to healthy control subjects. Given that cognitive impairment in FM is one of the emerging key symptoms, quantitative gait analysis under motor and cognitive dual tasks could point toward influence of cognitive changes on gait pattern. Furthermore, better understanding of gait changes might lead to better characterization of FM patients, and possibly could distinguish FM subgroups [11, 12].

Our objective was to assess quantitative spatiotemporal gait parameters in female FM patients in comparison to healthy controls during base walk and while performing motor, cognitive and combined, both motor and cognitive dual tasks. Further, aim was also to explore possible correlations of dual task gait pattern alterations to patients' functional status and presence or absence of clinical symptoms associated with FM.

\section{Methods}

\section{Patients}

Twenty-four female patients (mean age $55.08 \pm 11.28$ years) were consecutively recruited at the Institute of Rheumatology, Medical faculty, University of Belgrade, Serbia. Patients' diagnoses were based on revised diagnostic criteria for FM [5] and were regularly seen at the Institute for at least one year after the diagnosis was established. We recruited and examined 24 consecutive patients that fulfilled inclusion/exclusion criteria in the period of July-December 2019. Twentyfour healthy age and gender matched subjects (mean age $55.17 \pm 11.18$ years) served as a control group (HC). HC were recruited among healthy employees of the Medical Faculty, University of Belgrade. Subjects with conditions that could impair independent walking (neurological disorders, orthopedic diseases or other medical conditions, use of cane or walker) were not included. Additionally, patients with major psychiatric disorders, including major depression were not included in the study. All patients were on stable treatment for at least 2 months prior to inclusion in the study, both fibromyalgia treatment and use of antidepressant therapy, if any. The study was approved by Institutional Ethical Committee and written informed consent was obtained from each participant prior to the inclusion in this study. The study was performed in accordance with the ethical standards of the Declaration of Helsinki and its later amendments.

\section{Experimental protocol}

Gait parameters were measured using GAITRite electronic walkway of $5.5 \mathrm{~m}$ (CIR Systems, Havertown, PA) during base walk at preferred velocity and while performing dual motor, dual mental and combined tasks [20,21]. All participants performed six passes (three times down the pathway and back) at self-paced velocity, starting and ending their walks approximately $1.5 \mathrm{~m}$ before and after the walkway. Then they turn around and start their next pass. The motor dual task consisted of walking with a large ("pint") glass which was filled with water, with the aim of not spilling the water. The mental/cognitive dual task was serial "7" subtraction while walking, starting from randomly chosen numbers 90,95 or 100 . The combined motor and cognitive task comprised of serial subtractions as described, while walking with glass with water. The walking distance consisted of app $50 \mathrm{~m}$ (six passes $\times 8-9 \mathrm{~m}$ ) for each task (basic, motor, mental, and combined) which finally amounted to $200 \mathrm{~m}$ of walking, sufficient to assess gait variability $[20,21]$.

\section{Assessment of gait data}

We measured spatiotemporal parameters-cycle time $(\mathrm{CT})$, stride length (SL), swing time (SWT) and double support time (DST). Variability of gait parameters was calculated through the coefficient of variation $(\mathrm{CV})$ - variability normalized to mean value $(\mathrm{CV}=100 \times \mathrm{SD} /$ mean $)$ [22]. These parameters were chosen because of their association with executive functions, cognitive decline and/or gait rhythmicity in different patient and nonpatient populations $[6,7]$. Data from the activated sensors are collected and the GAITRite software calculated spatiotemporal parameters.

\section{Assessment questionnaires}

The following assessment questionnaires were used to estimate functional status of patients, pain and fatigue level, presence of depression, as well as cognitive functions status. The evaluator performing clinical assessments was a single, blinded, and experienced physician/ rheumatologist.

\section{Fibromyalgia Impact Questionnaire Revised (FIQR)}

The FIQR is the instrument for measuring functional status in FM patients. It includes 21 items that are answered on numeric rating scale of 0 -to-10, with 10 reflecting greatest impairment. The time frame is the 7 days, with the items distributed across three associated domains: "function" (9 items); "overall impact" (2 items); and "severity of symptoms" (10 items). The scoring system 
is as follows: the physical function domain (0-to-90) is divided by 3 , the overall impact domain $(0$-to- 20$)$ is not transformed, and the severity of symptoms domain (0-to$100)$ is divided by 2 [23].

\section{Krupp Fatigue Severity Score (FSS)}

Severity and impact of fatigue was measured by FSS [24]. Patients were asked to rate their level of agreement (toward seven) or disagreement (toward zero) with nine statements. A score of 36 and above (out of a maximum of 63) indicated the presence of significant fatigue. A higher score indicates greater fatigue. FSS is most effective as a measure of disability-related fatigue arising from a chronic, debilitating illness.

\section{Visual Analog Scale of pain intensity}

The visual analog scale (VAS) is a validated, subjective measure for acute and chronic pain. Scores are recorded by making a handwritten mark on a $10-\mathrm{cm}$ line that represents a continuum between "no pain" and "worst pain" (or more precisely between 0 and 100\%).

\section{The Short Form (36) Health Survey}

All patients completed the SF-36 questionnaire as a measure of health related quality of life [25]. The SF-36 is a generic instrument that measures eight general health concepts: physical functioning (PF), role physical $(\mathrm{RP})$, bodily pain $(\mathrm{BP})$, general health $(\mathrm{GH})$, vitality $(\mathrm{VT})$, social functioning (SF), role emotional (RE) and mental health $(\mathrm{MH})$. Two main scores are available to summarize these scales: physical composite score (PCS) and mental composite score (MCS), as well as total SF-36 score. All these scores fall within a $0-100$ scale, with higher scores reflecting better health related quality of life.

\section{Euroqol 5 dimensions (EQ 5D 3L)}

All patients completed the 3-level version of EuroQol 5 dimensions (EQ-5D-3L). The EQ-5D-3L essentially consists of 2 pages: the EQ-5D descriptive system and the EQ visual analogue scale (EQ VAS). The first part records the severity of the problem in each of the five dimensions, and the second part contains a $20-\mathrm{cm}$ vertical visual analogue scale ranging from 0 to 100 , where 0 corresponds to the worst imaginable health states and 100 corresponds to the best health states. Its five dimensions are mobility, self-care, usual activities, pain/discomfort, and anxiety/depression, and each dimension has three levels of severity (no problems, moderate problems, and extreme problems). Health status is labeled with a fivedigit number that represents the severity level in each dimension [26].

\section{Mini Mental State Examination (MMSE)}

The MMSE is an 11-question assessment tool to test domains-orientation of time and place, registration of 3 words, attention and calculation (recall of 3 words, language and visual construction). The test is scored from 0 to 30 , where higher scores indicate better cognitive performance, and a score below 24 suggests dementia [27].

\section{Hamilton Depression Rating Scale (HAM-D)}

This 17-item scale questioning the depressive complaints in the past week. The maximum score is 53 points. The scoring is as follows: $0-7$ points indicates "no depression", 8-15 points indicates "mild depression", 16-28 points indicates "moderate depression", 29 points and above indicates "severe depression" [28].

\section{Beck's Depression Inventory (BDI)}

Beck's Depression Inventory was used to assess the mood [29]. This index is a validated instrument which includes 21 items measuring depression. Scores above 13 are indicating the presence of depression and scores above 21 to indicate major depression.

\section{Statistical analysis}

Shapiro-Wilk test was used for data normality assessment. Friedman test was used to analyze statistical significance through motor, cognitive and combined tests input within groups (FM and $\mathrm{HC}$ ). The independent t-test was performed for CT, SL, SWT and DST (parametric variables) while Mann-Whitney test (non-parametric) were performed for CV CT, CV SL, CV SWT and CV DST while FM and $\mathrm{HC}$ walking during different conditions. According to data obtained by normality tests, we used Spearman's (rho) correlation coefficient.

The analyses were performed using SPSS 17.0 (SPSS, Chicago, IL), and p-values $<0.05$ were considered significant.

\section{Results}

We included 24 female FM patients (mean age $55.08 \pm 11.28$ years) and 24 healthy controls (mean age $55.17 \pm 11.18$ years). Demographic and clinical features of patients are presented in Table 1.

Patients were on average in the range of mild depression, according to HAM-D $(15.63 \pm 6.03)$ and BDI (14.63 \pm 6.36$)$. During evaluation, 11 patients (45.8\%) reported use of antidepressant therapy.

Gait parameters of FM patients and healthy control subjects (cycle time, stride length, swing time and double support time, and CVs of those parameters) were presented for all experimental conditions during base walk, 
Table 1 Demographic and Clinical features of fibromyalgia patients $(n=24)$

\begin{tabular}{ll}
\hline Value & Mean \pm SD \\
\hline Disease duration (years) & $11.72 \pm 8.59$ \\
Time from diagnosis (years) & $4.75 \pm 3.42$ \\
FIQR & $50.68 \pm 18.20$ \\
FSS & $4.77 \pm 1.65$ \\
VAS & $66.25 \pm 18.84$ \\
SF 36 PCS & $35.83 \pm 14.85$ \\
SF 36 MCS & $46.29 \pm 19.23$ \\
SF 36 total & $41.12 \pm 16.84$ \\
EQ 5D 3L & $62.92 \pm 22.55$ \\
MMSE & $29.00 \pm 1.35$ \\
HAM-D & $15.63 \pm 6.03$ \\
BDI & $14.63 \pm 6.36$ \\
\hline
\end{tabular}

Values present means \pm SDs_FIQR: Fibromyalgia Impact Questionnaire Revised; FSS: Krupp's Fatigue Severity Scale; VAS: visual analog scale; SF 36 PCS: The Short Form (36) Health Survey: physical composite score; SF 36 MCS: The Short Form (36) Health Survey - mental composite score; SF 36 total: The Short Form (36) Health Survey total score; EQ 5D 3L: Eurogol 5 dimensions 3-level version; HAMD: Hamilton Depression Rating Scale; MMSE: Mini Mental State Examination; BDI: Beck Depression Inventory

dual motor task, dual mental task and combined task in Fig. 1 (gait parameters on left panels, their CVs on right panels).

Assessing the impact of motor, cognitive and combined tasks in all subjects, FM patients and $\mathrm{HC}$, confirmed that these tasks affect gait performance (Friedman test). There were no differences in within group comparisons of FM patients and $\mathrm{HCs}$ in any of the assessed parameters, except in double support time during combined task, which was significantly prolonged in FM patients compared to HC (Fig. 1-" represents significance $p=0.022$ ).

Between group comparisons showed that cycle time of FM patients was significantly longer than in $\mathrm{HC}$ while stride length was shorter in patients for all four walking conditions. Swing time was prolonged in FM patients compared to $\mathrm{HC}$ for base and motor tasks, while double support time was significantly different for motor, mental and combined tasks (see Table 2, all significant differences are italic values). Further, note that CV's of all gait parameters were not significantly different in FM compared to $\mathrm{HC}$ in any of the walking conditions.

There was no correlation of VAS, FSS, MMSE, SF 36 physical, SF 36 total and FIQR with any of the four gait parameters and their CVs, performed in any of four walking tasks for FM patients. BDI correlate to cycle time, stride length and double support time while performing combined task $(\rho=0.58, p<0.01 ; \rho=-0.5, p<0.05$ and $\rho=0.58, p<0.01$, respectively). In FM patients HAM-D correlate to stride length and double support time. Correlations were also found in six other CVs of gait parameters through all four tasks (see details in Table 3). Concerning EQ 5D 3L, we found correlation for SWT and CV SWT and CV DST in most of the walking tasks, respectively. Correlations of SF 36 mental are also shown in Table 3.

\section{Discussion}

Study findings indicate that gait was impaired in FM patients, and gait performance correlates to clinical symptoms associated with fibromyalgia. Compared to their matched HC, FM female patients have shown significant differences in spatio-temporal gait parameters (CT, SL, SWT and DST) while their CV's were not significantly different in any of the walking conditions. Those findings were different to earlier literature findings [16-18].

In studies measuring gait while female FM patients performed several motor tasks $[14,15]$, or during base walking [17] and walking with different velocities [16, 17], results did not support gender-specific differences [16]. In general, women with FM have lower pain thresholds, greater diffuse pain, a higher number of tender points, higher levels of fatigue and greater sleep disturbance compared to men with FM [30-33]. However, differences in spatio-temporal gait variables were not reported [1619]. Therefore, our study with only female patients could give conclusions irrespective of patients' gender, while making patient group more coherent concerning gait characteristics.

Our patients were in the range of mild depression (Table 1). It was shown earlier that gait in patients with depression is affected in a certain manner [e.g., 20, 34]. In our earlier study with very similar protocol, patients with major depression demonstrated slower gait in all task conditions compared to $\mathrm{HC}$, while maintaining stability. Tasks that required greater cognitive involvement increased gait variability in patients, but only up to a certain level, after which patients' gait stability appeared unaffected by the increase of cognitive demand [20]. Gait in FM patients is affected similarly, showing similar pattern of gait parameters changes as described for patients with depressive disorder [20]. That could point toward important influence of depressive symptoms in FM on gait. Further, it is interesting that HAM-D scores negatively correlated to stride length in all conditions while were positively associated to DST only where cognitive input was engaged (i.e. mental and combined tasks). BDI correlated to CT, SL and DST only during combined, more complex tasks. It could be speculated that symptoms of depression influence patients to reduce stride length and double support time in comparison to their matched control subjects, such a measures to preserve stability of gait, mostly affected while cognitive, mental 


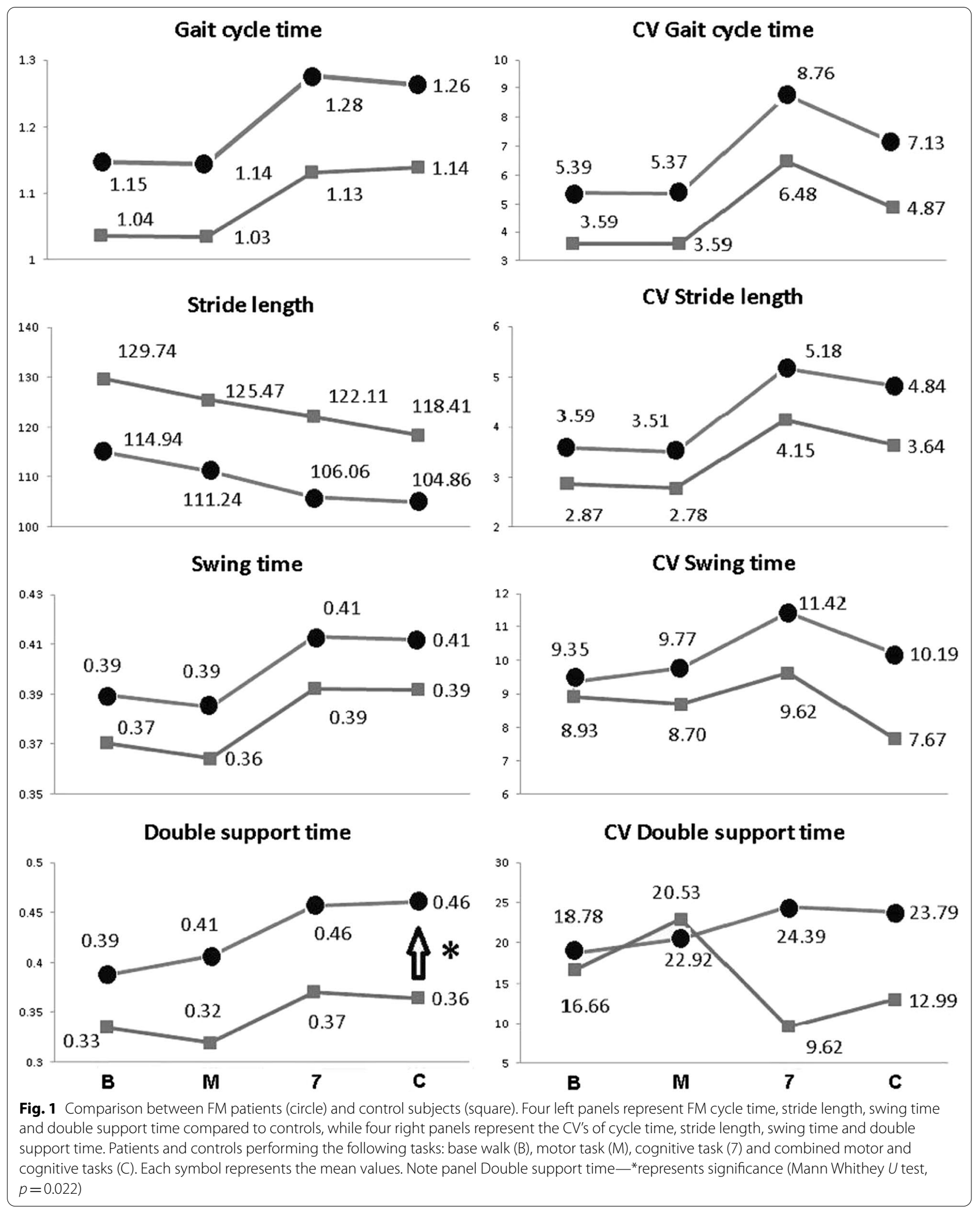


Table 2 Between group comparisons (FM vs HC) of gait parameters for all experimental conditions

\begin{tabular}{lllllllll}
\hline & CT & SL & SWT & DST & CV CT & CV SL & CV SWT & CV DST \\
\hline Base walk & 0.001 & 0.003 & 0.032 & 0.149 & 0.248 & 0.303 & 0.837 & 0.621 \\
Motor task & 0.001 & 0.003 & 0.041 & 0.039 & 0.103 & 0.103 & 0.398 & 0.711 \\
Mental task & 0.005 & 0.003 & 0.125 & 0.038 & 0.266 & 0.138 & 0.293 & 0.564 \\
Combined task & 0.006 & 0.009 & 0.123 & 0.014 & 0.278 & 0.120 & 0.090 & 0.334 \\
& $t$ test & & & \multicolumn{2}{c}{ Mann-Whitney test } \\
\hline
\end{tabular}

CT Cycle Time, SL Stride Length, SWT Swing Time, DST Double support time, CV Coefficient of variation

Table 3 Correlations of HAM-D, EQ-5D-3L and SF 36m to gait parameters in all walking tasks

\begin{tabular}{|c|c|c|c|c|c|c|c|c|}
\hline & CT & SL & SWT & DST & CV CT & CV SL & CV SWT & CV DST \\
\hline \multicolumn{9}{|l|}{ HAM-D } \\
\hline Base walk & 0.13 & $-0.4^{*}$ & -0.34 & 0.35 & $0.49^{*}$ & $0.46^{*}$ & 0.29 & 0.21 \\
\hline Motor task & 0.39 & $-0.42^{*}$ & -0.34 & 0.36 & $0.48^{*}$ & 0.29 & 0.32 & 0.15 \\
\hline Mental task & 0.35 & $-0.5^{*}$ & -0.11 & $0.51^{*}$ & 0.26 & 0.36 & $0.58^{* *}$ & $0.42^{*}$ \\
\hline Combined task & 0.41 & $-0.58^{* *}$ & 0.20 & $0.69^{* *}$ & 0.32 & 0.32 & $0.58^{* *}$ & 0.31 \\
\hline \multicolumn{9}{|l|}{ EQ 5D $3 L$} \\
\hline Base walk & 0.19 & 0.06 & $0.57^{* *}$ & -0.20 & -0.27 & -0.04 & $0.44^{*}$ & $-0.59^{* *}$ \\
\hline Motor task & 0.28 & 0.07 & $0.49^{*}$ & -0.15 & -0.40 & -0.01 & $-0.43^{*}$ & $-0.45^{*}$ \\
\hline Mental task & 0.12 & 0.13 & 0.32 & -0.11 & -0.21 & -0.13 & $-0.61^{* *}$ & $0.5^{*}$ \\
\hline Combined task & -0.05 & -0.27 & $0.49^{*}$ & -0.30 & -0.21 & -0.13 & $-0.55^{*}$ & $-0.48^{*}$ \\
\hline \multicolumn{9}{|l|}{ SF $36 m$} \\
\hline Base walk & 0.05 & 0.35 & 0.28 & -0.19 & -0.40 & -0.33 & -0.25 & -0.34 \\
\hline Motor task & -0.07 & 0.32 & 0.18 & -0.14 & $-0.43^{*}$ & -0.18 & -0.23 & -0.23 \\
\hline Mental task & -0.16 & 0.38 & 0.2 & -0.25 & -0.36 & -0.37 & $-0.53^{* *}$ & $-0.42^{*}$ \\
\hline Combined task & -0.41 & $0.55^{*}$ & 0.18 & $-0.55^{*}$ & -0.35 & -0.41 & $-0.46^{*}$ & -0.31 \\
\hline
\end{tabular}

HAM-D: Hamilton Depression Rating Scale; EQ 5D 3L: Euroqol 5 dimensions 3-level version; SF 36m: The Health Survey Mental composite score. Correlations of other used assessment questionnaires and gait parameters were described in Result section.

$\rho($ rho $)=$ Spearman correlation, ${ }^{*} p<0.05,{ }^{* *} p<0.01$

tasks requirements were employed. That might prevent negative cognitive input on gait stability, shortening the strides and increasing time with both feet on the ground, reducing gait variability in such complex situations. However, our patients were not suffering from major depression and were on stable antidepressant treatment, suggesting possible impact of FM per se.

Patients' gait is examined under dual task conditions, and possible impairments in dual task walking are estimated in relation to mental/cognitive and motor engagement. CT and SL were different between FM and HC for all experimental conditions (Table 2). Further, SWT was different in base and motor tasks, while it was not different during mental and combined tasks. That finding could mean that FM patients behave differently from $\mathrm{HC}$, and during tasks without cognitive employment, their gait pattern is changed. The parameters affected by base walk or motor performance might be linked to clinical symptoms associated with FM, such as presence of chronic pain, bradykinesia, stiffness or fatigue [13$15,17]$, and that could be responsible for gait alterations. Further, tasks with cognitive inputs (mental and combined) show no significant influence. That could be explained by importance and strong input of cognition on gait, on both groups, where $\mathrm{HC}$ also change their gait pattern while performing a secondary mental task [e.g., 7, 35, 36] (Table 2, Fig. 1). Additional tasks did not significantly affect described gait parameters changes, changes are already present, to preserve stability. Additionally, FM patients had considerable prolongation of DST while performing combined task (Table 2 and Fig. 1), stressing certain level of postural instability [37]. Since swing time and double support time were reported to reflect posture and balance [7, 21, 37], that could be the action to increase stability due to increased attention and awareness of patients of requirement to fulfill the complex given tasks. On the other hand, gait variability was not significantly increased as earlier described in the study 
where FM females were tested [17], but trend of greater CVs across all tasks was observed that did not reach statistical significance, possibly due to smaller sample in our study. Alternatively, our patients tend to preserve stability in those dual tasks, complex walking situations.

In order to assess the influence of the disease clinical characteristics, we correlated gait parameters in all experimental conditions to the questionnaires and patients' reports. There were no significant correlation of VAS, FSS, MMSE, SF 36 physical, SF 36 total and FIQR with any of the four gait parameters and their CVs, performed in any of four walking tasks for FM patients. Correlates of depression status (BDI and HAM-D) are particularly present for stride length, and that was discussed earlier. Correlations of EQ 5D 3L and SF 36m-severity of the health status and quality of life in the domain of mental health with evaluated gait parameters are shown in Table 3. Most of the correlations were with the parameters SWT, CV of SWT and DST. As it was said earlier, swing time and double support time were reported to reflect posture and balance, possibly as a function of preserved central mechanisms [7, 21, 37]. Therefore our patients preserve gait stability and adapt gait pattern for complex requirements while walking, probably because of their perceived deteriorated health status and its mental component that could change gait and evoke walking instability. Attention should be paid to depression status of FM patients in order to either improve gait pattern or to use that tests to define FM subgroup and focus on treatment of depression. Same is true for assessment of severity of the perceived health status (e.g. EQ 5D 3L) and quality of life patients' experiencing, especially in the domain of mental health (e.g. SF 36m).

Limitation of the study was the design where we performed single center, observational, cross-sectional study and we could suggest that longitudinal studies would strengthen the study findings and recommendations. Also, lower prevalence of FM in man limited us to perform study in female patients only. Finally, the number of our female patients took antidepressant medication and, therefore, the effect of this medication on gait characteristics could not be completely neglected.

\section{Conclusions}

It could be concluded that gait is affected in FM patients during dual walking tasks. Cognitive tasks, alone or combined with motor task, had a larger effect than motor tasks. However, no changes in stride-to-stride variability indicated that patients preserve stability in complex walking situations, in spite of potential clinical symptoms effect. Therefore, analysis of gait may provide additional information for the FM identification based on presence of certain clinical symptoms and cognitive status. Attention also should be paid to depression status of patients to either improve gait pattern or to use that tests to define FM subgroups. According to these results we could further support the approach that correlation of dual task gait pattern alterations to existence of FM symptoms and cognitive changes influencing gait pattern could successfully define FM subgroups.

\section{Acknowledgements}

The study has been supported by the Ministry of Education, Science and Technological Development, Republic of Serbia.

\section{Authors' information}

GR, ZV, MR and SJ are rheumatologists, experienced in fibromyalgia treatment and research. VM and SR are neurologists, experienced in gait research.

\section{Authors' contributions}

All listed authors participated significantly in this study. Study aim creation, conceptualization and design: GR and SR. Acquisition of data-recordings of patients and control subjects: SR, ZV, MR and SJ. Analysis and interpretation of data: GR, ZV, VM and SR. Drafting of the manuscript: SR, VM and GR. Critical revision of the manuscript for important intellectual content: SR. Statistical analysis: GR, VM. Study supervision and final approval: SR and GR. All authors read and approved the final manuscript.

\section{Funding}

The study has been supported by the Ministry of Education, Science and Technological Development, Republic of Serbia.

\section{Availability of data and materials}

The datasets used and/or analysed during the current study are available from the corresponding author on reasonable request.

\section{Declarations}

Ethics approval and consent to participate

The study was approved by Institutional Ethical Committee and written informed consent was obtained from each participant prior to the inclusion in this study. The study was performed in accordance with the ethical standards of the Declaration of Helsinki and its later amendments.

Consent for publication

Not applicable.

\section{Competing interests}

The authors declare that they have no competing interests.

\section{Author details}

${ }^{1}$ Institute of Rheumatology, Faculty of Medicine, University of Belgrade, Resavska 69, Belgrade, Serbia. ${ }^{2}$ Neurology Clinic, Faculty of Medicine, University of Belgrade, Dr Subotića 6, Belgrade, Serbia. ${ }^{3}$ Institute for Medical Research, University of Belgrade, Dr Subotića 4, 11129 Belgrade, Serbia.

Received: 18 May 2021 Accepted: 18 August 2021

Published online: 26 August 2021

References

1. Borchers AT, Gershwin ME. Fibromyalgia: a critical and comprehensive review. Clin Rev Allergy Immunol. 2015;49:100-51.

2. Clauw DJ. Fibromyalgia and related conditions. Mayo Clin Proc. 2015;90:680-92.

3. Wolfe F, Clauw DJ, Fitzcharles MA, Goldenberg DL, Katz RS, Mease P, et al. The American College of Rheumatology preliminary diagnostic criteria for fibromyalgia and measurement of symptom severity. Arthritis Care Res (Hoboken). 2010;62:600-10. 
4. Wolfe F, Clauw DJ, Fitzcharles MA, Goldenberg DL, Häuser W, Katz RS, et al. Fibromyalgia criteria and severity scales for clinical and epidemiological studies: a modification of the ACR Preliminary Diagnostic Criteria for Fibromyalgia. J Rheumatol. 2011;38:1113-22.

5. Wolfe F, Clauw DJ, Fitzcharles MA, Goldenberg DL, Katz RS, Mease P, et al. Revisions to the 2010/2011 fibromyalgia diagnostic criteria. Semin Arthritis Rheum. 2016:46:319-29.

6. Yogev G, Giladi N, Peretz C, Springer S, Simon ES, Hausdorff JM. Dual tasking, gait rhythmicity, and Parkinson's disease: which aspects of gait are attention demanding? Eur J Neurosci. 2005;22:1248-56.

7. Yogev-Seligmann G, Hausdorff JM, Giladi N. The role of executive function and attention in gait. Mov Disord. 2008;23:329-42.

8. Giesecke T, Williams DA, Harris RE, Cupps TR, Tian X, Tian TX, et al. Subgrouping of fibromyalgia patients on the basis of pressure-pain thresholds and psychological factors. Arthritis Rheum. 2003;48:2916-22.

9. Wilson HD, Robinson JP, Turk DC. Toward the identification of symptom patterns in people with fibromyalgia. Arthritis Rheum. 2009;61:527-34.

10. Gracely RH, Ambrose KR. Neuroimaging of fibromyalgia. Best Pract Res Clin Rheumatol. 2011;25:271-84.

11. Auvinet $B$, Chaleil D, Cabane J, Dumolard A, Hatron P, Juvin R, et al. The interest of gait markers in the identification of subgroups among fibromyalgia patients. BMC Musculoskelet Disord. 2011;12:258.

12. Auvinet $B$, Chaleil $D$. Identification of subgroups among fibromyalgia patients. Reumatismo. 2012;64:250-60.

13. Auvinet B, Bileckot R, Alix AS, Chaleil D, Barrey E. Gait disorders in patients with fibromyalgia. Jt Bone Spine. 2006;73:543-6.

14. Costa ID, Gamundí A, Miranda JG, França LG, De Santana CN, Montoya P. Altered functional performance in patients with fibromyalgia. Front Hum Neurosci. 2017;11:14.

15. Martín-Martínez JP, Villafaina S, Collado-Mateo D, Fuentes-García JP, PérezGómez J, Gusi N. Impact of cognitive tasks on biomechanical and kinematic parameters of gait in women with fibromyalgia: a cross-sectional study. Physiol Behav. 2020;227:113171

16. Heredia-Jimenez J, Orantes-Gonzalez E. Gender differences in patients with fibromyalgia: a gait analysis. Clin Rheumatol. 2019;38:513-22.

17. Heredia-Jimenez J, Orantes-Gonzalez E, Soto-Hermoso VM. Variability of gait, bilateral coordination, and asymmetry in women with fibromyalgia. Gait Posture. 2016:45:41-4.

18. Heredia-Jiménez JM, Aparicio García-Molina VA, Porres Foulquie JM, Delgado Fernández M, Soto-Hermoso VM. Spatial-temporal parameters of gait in women with fibromyalgia. Clin Rheumatol. 2009;28:595-8.

19. Heredia-Jimenez JM, Soto-Hermoso VM. Kinematics gait disorder in men with fibromyalgia. Rheumatol Int. 2014;34:63-5.

20. Radovanović S, Jovičić M, Marić NP, Kostić V. Gait characteristics in patients with major depression performing cognitive and motor tasks while walking. Psychiatry Res. 2014;217:39-46.

21. Radovanović S, Perić S, Savić-Pavićević D, Dobričić V, Pešović J, Kostić $\checkmark$, et al. Comparison of temporal and stride characteristics in myotonic dystrophies type 1 and 2 during dual-task walking. Gait Posture. 2016:44:194-9.
22. Menz HB, Latt MD, Tiedemann A, MunSanKwan M, Lord SR. Reliability of the GAITRite walkway system for the quantification of temporo-spatial parameters of gait in young and older people. Gait Posture. 2004;20:20-5.

23. Bennett RM, Friend R, Jones KD, Ward R, Han BK, Ross RL. The Revised Fibromyalgia Impact Questionnaire (FIQR): validation and psychometric properties. Arthritis Res Ther. 2009;11:415.

24. Krupp LB, LaRocca NG, Muir-Nash J, Steinberg AD. The fatigue severity scale. Application to patients with multiple sclerosis and systemic lupus erythematosus. Arch Neurol. 1989:46:1121-3.

25. Beyazal MS, Tüfekçi A, Kırbaş S, Topaloğlu MS. The impact of fibromyalgia on disability, anxiety, depression, sleep disturbance, and quality of life in patients with migraine. Noro Psikiyatr Ars. 2018;55:140-5.

26. Collado-Mateo D, Dominguez-Muñoz FJ, Adsuar JC, Garcia-Gordillo MA, Gusi N. Effects of exergames on quality of life, pain, and disease effect in women with fibromyalgia: a randomized controlled trial. Arch Phys Med Rehabil. 2017:98:1725-31.

27. Folstein MF, Folstein SE, McHugh PR. "Mini-mental state". A practical method for grading the cognitive state of patients for the clinician. J Psychiatr Res. 1975;12:189-98.

28. Hamilton M. A rating scale for depression. J Neurol Neurosurg Psychiatry. 1960;23:56-62.

29. Beck AT, Ward CH, Mendelson M, Mock J, Erbaugh J. An inventory for measuring depression. Arch Gen Psychiatry. 1961;4:561-71.

30. Wolfe F, Ross K, Anderson J, Russell IJ. Aspects of fibromyalgia in the general population: sex, pain threshold, and fibromyalgia symptoms. J Rheumatol. 1995;22:151-6.

31. Yunus MB, Celiker R, Aldag JC. Fibromyalgia in men: comparison of psychological features with women. J Rheumatol. 2004;31:2464-7.

32. Miró E, Martínez MP, Sánchez Al, Prados G, Lupiáñez J. Men and women with fibromyalgia: relation between attentional function and clinical symptoms. Br J Health Psychol. 2015;20:632-47.

33. Yunus MB, Inanici F, Aldag JC, Mangold RF. Fibromyalgia in men: comparison of clinical features with women. J Rheumatol. 2000;27:485-90.

34. Lemke MR, WendorffT, Mieth B, Buhl K, Linnemann M. Spatiotemporal gait patterns during over ground locomotion in major depression compared with healthy controls. J Psychiatr Res. 2000;34:277-83.

35. Kressig RW, Beauchet O, European GAITRite Network Group. Guidelines for clinical applications of spatio-temporal gait analysis in older adults. Aging Clin Exp Res. 2006;18:174-6.

36. Hausdorff JM, Edelberg HK, Mitchell SL, Goldberger AL, Wei JY. Increased gait unsteadiness in community-dwelling elderly fallers. Arch Phys Med Rehabil. 1997;78:278-83.

37. Marchese R, Bove M, Abbruzzese G. Effect of cognitive and motor tasks on postural stability in Parkinson's disease: a posturographic study. Mov Disord. 2003;18:652-8.

\section{Publisher's Note}

Springer Nature remains neutral with regard to jurisdictional claims in published maps and institutional affiliations.

\footnotetext{
Ready to submit your research? Choose BMC and benefit from:

- fast, convenient online submission

- thorough peer review by experienced researchers in your field

- rapid publication on acceptance

- support for research data, including large and complex data types

- gold Open Access which fosters wider collaboration and increased citations

- maximum visibility for your research: over $100 \mathrm{M}$ website views per year
}

At BMC, research is always in progress.

Learn more biomedcentral.com/submissions 